\title{
The effects of learning instructions and cueing on free recall*
}

\author{
FRANCES L. PRESTIANNI \\ Temple University. Philadelphia, Pennsylvania 19122 \\ and \\ ROSE T. ZACKS \\ Michigan State University. East Lansing, Michigan 48823
}

\begin{abstract}
Eighty unrelated high-imagery words were presented for free recall learning in groups of four words each. Learning instructions were varied, with one group told to relate the words in each input group by means of images, another group told to use verbal (sentence) mediators, and the control group not given specific learning instructions. During recall, list items were presented as retrieval cues in some conditions. In comparison with the control condition, imagery and verbal mediation instructions produced greater recall, more clustering, and a larger number of instances per group recalled. When the retrieval cues were selected such that one cue came from each of the input groups and when the strength of within-group organization was quite high, Ss recalled more words and a larger number of input groups. It was concluded that instructions and cueing conditions influenced different aspects of the free recall process. Instructions influenced the organization of individual words into units, and cueing affected the retrieval of specific units.
\end{abstract}

Free recall learning is often regarded as involving two distinct levels of organization: the clustering of individual items into groups (higher-order units, chunks) and the development of a retrieval plan that allows $S$ to move from one group to another during recall (e.g., Bower, 1970b; Tulving \& Pearlstone, 1966). The present study addresses itself simultaneously to both kinds of organizational processes. It thus provides information on possible interactions between the development of within-group organization and the development of the retrieval plan, as well as further data on variables which affect each of these organizational processes individually.

Various kinds of experimental manipulations have been used in attempts to influence within-group organization including variation of list construction, of order of item presentation, and of learning instructions. Learning instructions were varied in the present experiment. In order to have control over the groupings which Ss used, the list of 80 high-imagery words was presented in groups of 4 words each (cf. Bower, Lesgold, \& Tieman, 1969). One experimental group was told to use images to relate the 4 words in each group. A second experimental group was told to use verbal (sentence) mediators, and the control group was not given specific learning instructions. These kinds of learning instructions were chosen because of the current interest in comparing verbal and imaginal memory codes and because little of the work on this issue has involved tasks other than paired-associate learning (Paivio, 1971). In the paired-associate task, when care is taken to ensure that Ss use the kind of mediators they are instructed to

*The authors would like to thank Robert W. Weisberg, Lynn Hasher, and Gordon Wood for their comments on earlier versions of this paper. Requests for reprints should be sent to Rose T. Zacks, Department of Psychology, Michigan State University, East Lansing, Michigan 48823. use, it appears that imaginal mediators are superior to verbal mediators for high-imagery words (Paivio \& Foth, 1970: Bower \& Winzenz, 1970). This is an important finding for Paivio's (1971) dual-coding hypothesis, and it also attests to the great effectiveness of imaginal memory codes. However, further work needs to be done before it can be concluded that the same result holds for free recall learning. Very few free recall experiments have systematically compared imagery mediation. verbal mediation, and control conditions. One such study (Mueller \& Jablonski, 1970) found that imagery instructions facilitated performance relative to a no-mediation control condition, whereas sentence mediation instructions did not consistently do so. The present investigation provides further data on this point.

Cueing at recall was employed to study the retrieval process. In the main experimental conditions, one word from each of the input groups served as retrieval cues at recall. Judging from previous work on cueing in free recall, our best guess would have to be that this condition would not show better performance than a no-cue control condition, because the list consisted of unrelated words. When the list is categorized, category names generally facilitate recall by increasing the number of categories recalled (Tulving \& Pearlstone, 1966; Hudson \& Austin, 1970). Under some conditions intralist items are also effective cues, but only when the list is categorized or contains groups of associatively or semantically related words (Allen, 1969: Hudson \& Austin, 1970: Slamecka, 1972; Wood, 1969). Previous studies have found intralist cues not to be effective when the list consists of unrelated words (Allen. 1969; Slamecka, 1968, 1969).

Since the effects of cueing have important implications for the organizational account of free recall learning and for theorizing about the retrieval process (Postman, 1972). this matter would seem to warrant 
further study. Various reasons have been offered for negative findings in the cueing studies that have used lists of unrelated words by Allen (1969). Postman (1972). Wood (1969). and others. On the basis of these discussions and a survey of the cueing studies. it appears that for cues to facilitate recall a number of conditions must be met: (a) the within-chunk organization must be quite high. (b) there must be more chunks than $\mathrm{S}$ can recall unaided, and (c) the list organization and the relation of the cues to the list organization must be obvious to $\mathrm{S}$. The experiments using categorized lists have generally met these conditions and have found cueing to be beneficial. When these conditions are not met, cueing will not be effective even with a categorized list. as in Slamecka's (1968) Experiment IV which included only five categories. An attempt was made to meet the above conditions in the present experiment. and thus to show that a beneficial effect of cueing can occur with unrelated words.

Finally, the current research involved an independent groups design in which instructions and cueing were factorially combined. All groups had three presentation-recall trials on the same list. Thus. we can observe how the effects of instructions and cueing interact with each other and how these effects develop over trials.

\section{METHOD}

\section{Materials}

Euhty high-imagery nouns fratings of 6 or above on a 7 -point sale) were selected from norms compiled by Paivio. Yuille. and Madigan (1968). As far as was possible. Hords were chosen anong which there were no obvious relationships. The 80 words were randomly divided into 20 groups of 4 stimuli, with the restrition that no initial letter was repeated within a group. There were three presentation-recall trials with a different order of the 4-word input groups on each trial. However. the withingroup order was constant across the three trials.

\section{Design}

The design was a 3 by 3 factoral with an independent group of Ss receiving each treatment combination. One independent variable was instruction condition. and the three types of learning instructions were:

\section{lmager Condinon}

For each presentation group. the Ss were told to try to form a picture of the four objects interacting in some wat and were given an example of how this could be done.

\section{Ierbal Condition}

The same general form of instructions was used except that Ss were told to form a sentence containing the four words interacting in some way and were given an example using the sume words as in the imagery condition.

\section{Control Condition}

The instructions merely stated that words would be prevented in groups of four. and the same four words were viven as an '`ample.
The second independent variable was cueing condition, and one-third of the Ss in each instruction condition was randomly assigned to each of the following three cueing groups:

\section{0-Group Cues Condition}

On each recall trial a set of 20 cues was presented. The cues were list members, and each cue came from a different presentation group. A new set of cues was used on each trial, with none of the words serving as cues more than once. The order of presentation of the three sets of cues was randomized across Ss.

\section{Group Cues Condition}

Again, a different set of 20 list items was used as recall cues on each trial, and the order of presentation of the cue sets varied across Ss. This time, however, the 20 cues in a set came from only five presentation groups and completely exhausted these groups. This condition provided a control for the time required to read the list of cues in the 20-group cues condition.

\section{No Cues Condition}

No cues were presented during recall.

\section{Procedure}

Learning instructions were given orally, and the stimuli were shown by means of a slide projector with an 11 -sec exposure time for each 4-word group. After presentation of the 20 slides. $\mathrm{S}$ opened the answer booklet and was allowed $2 \mathrm{~min}$ to read the recall instructions. These instructions stated that all the words which $S$ could remember should be written in any order in the numbered blanks on the following page. For the two conditions receiving recall cues, the instructions also stated that 20 words from the presentation list would be given and that these might aid in recalling the other words. These cues were presented in a column at the right of the answer sheet. For the 20-group cues condition. the order of the cues was completely random. For the 5-group cues condition, the order of the 4-word groups was also random. but the within-group order was the same as during the presentation trial. All Ss were allowed $5 \mathrm{~min}$ to recall the words and then turned to a blank page in the recall booklet. The three study-test trials followed in immediate succession with oral input instructions and written recall instructions repeated on each trial.

\section{Subjects}

Two hundred and sixteen students in six undergraduate psychology classes at Temple Lniversity were tested during regular class periods. The three instruction conditions were each presented to two entire classes with each recall condition randomiy assigned to approximately one-third of the $S$ s within each class. The size of the classes varied from 35 to 45 . and students were randomly discarded to form subgroups of $20 \mathrm{Ss}$ in each of the nine experimental conditions for a total of $180 \mathrm{Ss}$.

\section{RESULTS}

\section{Dependent Measures}

The major dependent variable was the number of words recalled. Recall in the 20-group cues and 5-group cues conditions was scored only for the 60 nonpresented words. This made the maximum possible recall scores different in the three cueing conditions. and it was 
Fig. 1. Mean percentage of words recalled for instruction and cueing conditions across trials.

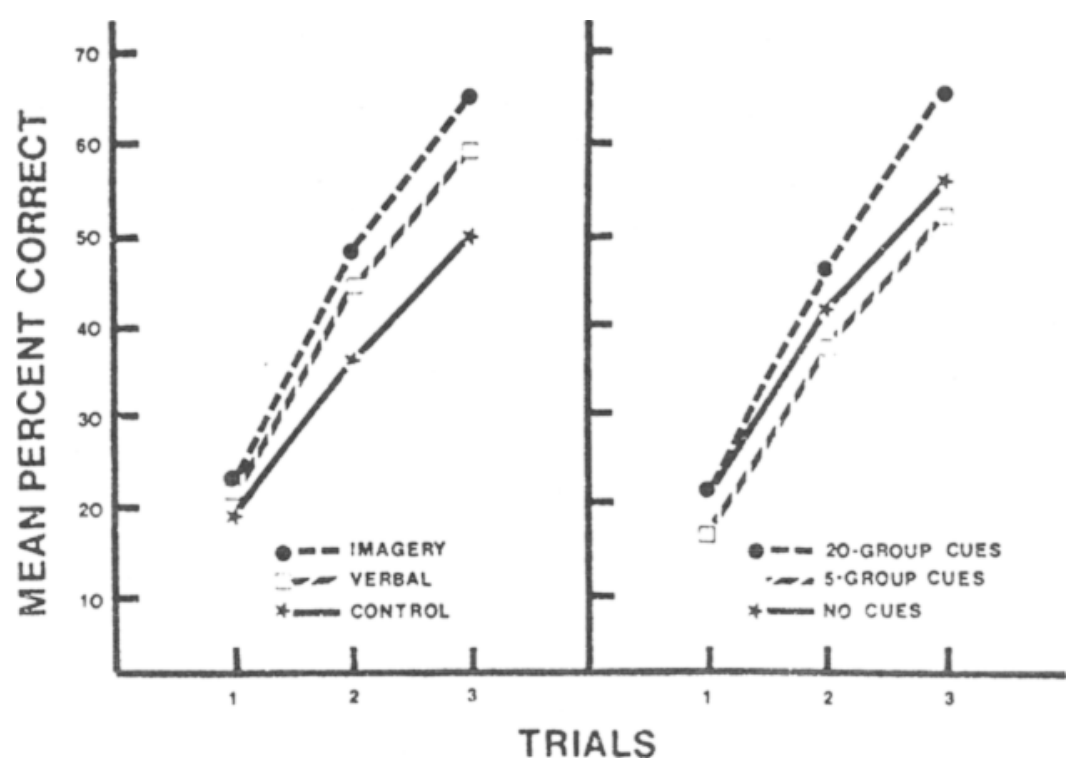

function of instruction condition and trials; the right panel shows word recall as a function of cueing condition and trials. It can be seen that both the instruction and the cueing variable influenced word recall. and the effects of both variables tended to increase over trials. Analysis of variance corroborates these observations in showing significant main effects for instruction condition $[F(2.171)=9.21 . p<01 . \mathrm{SE}=$ $.21]$. cueing condition $[F(2.171)=7.31, \mathrm{p}<.01, \mathrm{SE}=$ $.19]$, and trials $[F(2,342)=1,059.33, p<.01 . S E=$ 42]. The Instruction by Trials interaction $[F(4,342)=$ 14.33, $\mathrm{p}<.01, \mathrm{SE}=.08]$ and the Cueing by Trials interaction $[F(4,342)=9.00, p<.01, S E=.07]$ were also significant.

On all three trials. recall is highest for the imagery group, next best for the verbal group, and poorest for the control group. Tests of simple effects at each trial showed that reliable differences among the instruction conditions emerged at Trial 2. On this trial. Newman-Keuls tests indicated that the imagery and verbal groups did not differ from each other, but both were superior to the control group $(p<.01)$. According to Newman-Keuls tests, the difference between the imagery and verbal groups was significant on the third trial $(p<.05)$, as was the difference between each of these groups and the control group $(p<.01)$.

With regard to the cueing variable, it can be seen in the right panel of Fig. 1 that performance in the 20 -group cues condition was higher than in the no cues condition, while performance in the 5-group cues condition was lower than in the no cues condition. ${ }^{2}$ According to tests of simple effects at each trial, significant differences among the cueing conditions occurred on Trials 2 and 3 . On the second trial, the 20-group cues and 5-group cues conditions were reliably different ( $p<.01$ according to Newman-Keuls tests). On the third trial. the 20-group cues condition was reliably superior to both the 5-group cues and the no cues
The left panel of Fig. 1 presents word recall as a

\section{Words Recalled}




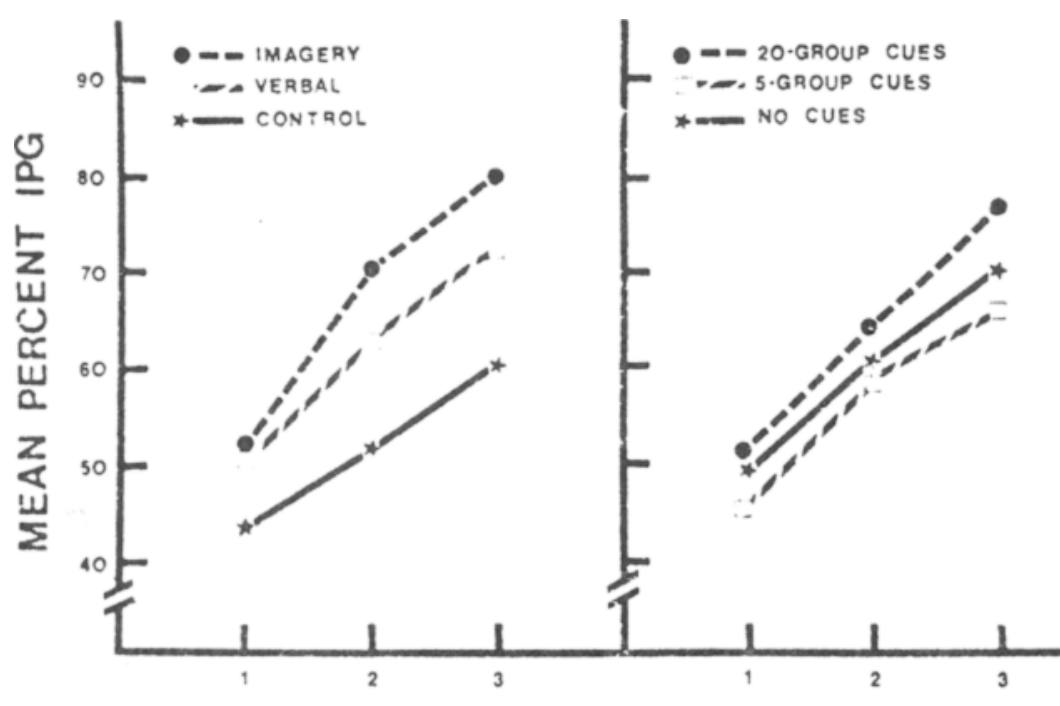

TRIALS
Fig. 2. Mean percentage of IPG for instruction and cueing conditions across trials. conditions $(\mathrm{p}<.01)$. Therefore, on the last trial at least. a beneficial effect of cueing was obtained in the 20.group cues condition.

Thus. telling Ss to relate the four words in a group by using a verbal or an image mediator (especially the latter) increased recall. Providing one word from each group as a recall cue also facilitated recall. The following analyses provide information on the locus of the effects of each of the independent variables (within-group organization vs the retrieval process).

\section{Within-Group Organization}

Both the ARC and IPG measures were strongly influenced by instruction condition and little affected by cueing condition. The mean clustering scores were 76. .63 . and .43 for the imagery. verbal, and control groups. These differences which mirror the differences in recall as a function of instruction condition were significant $[F(2.171)=29.89 . p<.01, \mathrm{SE}=.30]$. The only other reliable difference in the analysis of the $A R C$ scores was that for trials $[F(2.342)=6.29, p<.01 . S E=$ .05]. Clustering increased across trials with the increase between Trials 1 and 2 accounting for virtually all of the varance (means $=.55 .63$. and .64 ).

The IPG data are somewhat more complicated than the clustering data. The left panel of Fig. 2 presents IPG scores as a function of instruction condition and trials. The IPG scores were highest for imagery condition and lowest for the control condition. with the verbal condition falling in between but closer to the imagery group. The effects of cueing condition on the IPG scores are shown in the right panel of Fig. 2. Here the differences are much smaller. but the order of the groups from highest to lowest is consistently 20-group cues. no cues. and 5-group ties. As for the clustering data. analysis of variance of the IPG scores showed significant main effects for instruction condition $[F(2,171)=$ 20.71. $\mathrm{p}<.01, \mathrm{SE}=.33]$ and trials $[\mathrm{F}(2,342)=300.50$, $p<.01 . S E=.261$. The Instruction by Trials interaction $[\mathrm{F}(4.342)=13.25, \mathrm{p}<.01, \mathrm{SE}=.09]$ was also reliable and reflects the increasing divergence among the instruction conditions across trials.

While the main effect of cueing condition was not reliable, the Cueing by Triais interaction was significant $[F(4.342)=3.75 . p<.01 . S E=.05]$. Thus. cueing condition did have a small effect on within-group organization as measured by IPG, but the effect emerged only late in acquisition. It is not clear how much emphasis should be placed on this interaction. The other measure of within-group organization was not similarly affected by cueing condition. Also. the influence of instruction condition on IPG scores was much stronger than that of cueing manipulation. The maximum difference in IPG scores as a function of instruction condition was $20 \%$ (the imagery-control difference on the third trial). while the maximum difference in IPG scores as a function of cueing condition was only $10 \%$ (the 20-group cues/5-group cues difference on the third trial). Further. it is possible that the cueing condition affected the IPG measure not directly. but rather because the 20-group cues condition allowed Ss to devote more time to strengthening within-group organization relative to the other cueing conditions. $\mathrm{By}$ providing access to each input group. the 20 -group cues condition allowed $\mathrm{S}$ to devote relatively little effort to developing a retrieval plan: it is possible that Ss became increasingly aware of this over trials.

\section{Groups Recalled}

The NGR data for the three cueing conditions are shown in Fig. 3. The number of groups recalled is consistantly howest in the 5-group cues condition. On 
the second and third trials. the NGR scores are highest for the 20-group cues condition. with the no cues condition in the middle. These effects of cueing on NGR scores are similar to the effects of cueing on word recall. The analysis of variance for the NGR data yielded significant main effects for cueing condition $[F(2,171)=$ 4.72. $p<.01, \mathrm{SE}=.15]$ and trials $[\mathrm{F}(2.342)=636.50$, $\mathrm{p}<.01, \mathrm{SE}=.46]$. The significant Cueing by Trials interaction $[F(4.342)=2.67, p<.01, S E=.05]$ is consistent with the argument that for the 20-group cues condition to facilitate group retrievability, a high level of within-group organization is required, and this was apparently not achieved in the present case until the second or third trial.

The only significant comparison involving instruction condition for the NGR scores was the Instruction by Cueing interaction $[F(4.171)=4.00, p<.01, S E=.24]$. This interaction requires some comment. It reflects differing effects of cueing condition depending on the particular instruction condition. The relevant means are shown in Table 1. For Ss who learned under imagery instructions, the trend of the data is such that the 20.group cues condition resulted in increased accessibility of the input groups and the 5-group cues condition resulted in decreased accessibility relative to the no cues condition. The imagery instructions produced the highest within-group organization, and the effects of cueing are similar to ones expected with

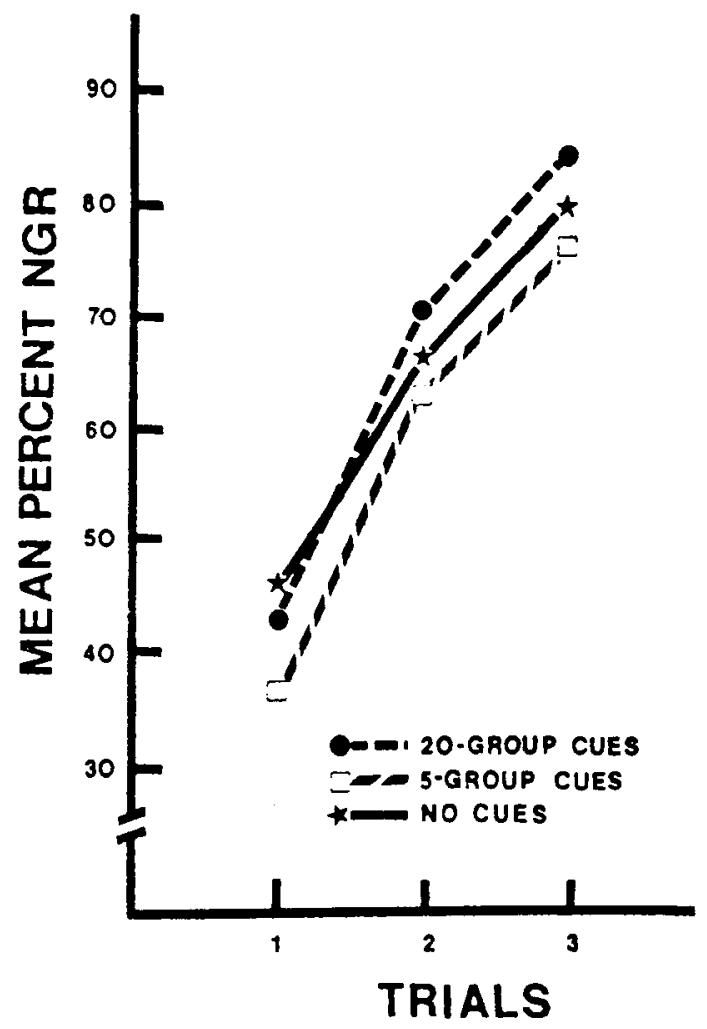

Fig. 3. Mean percentage of NGR for cueing conditions across trials.
Table 1

Mean Percentage of NGR for Each of the Groups

\begin{tabular}{lccc}
\hline & Imagery & Verbal & Control \\
\hline 20-Group Cues & 71 & 66 & 61 \\
No Cues & 61 & 63 & 69 \\
5-Group Cues & 55 & 61 & 63 \\
\hline
\end{tabular}

categorized lists. The pattern of NGR data for the verbal group is similar to that for the imagery group, but the differences are much reduced, presumably because within-group organization was not quite so high. For the control group, the 20-group cues condition was, if anything, interfering. The control group results look like previous data on the effects of intralist cueing with lists of unrelated words. If this interpretation is correct, then the instruction variable did not have a direct effect on group accessibility, but rather had an indirect effect via its influence on within-group organization.

\section{Summary of Results}

The results of this experiment can be summarized in terms of the two kinds of organizational factors involved in free recall learning. These are the strength of within-group organization and the accessibility of the higher-order groups, and total recall depends on both these factors. The main locus of effect of the instruction variable was on the first factor, the strength of within-group organization. Imagery and verbal instructions increased within-group cohesiveness. In contrast, the cueing conditions were most effective in determining the accessibility of the higher-order groups.

There are several lines of evidence which support this organization of the data: (a) The number of words recalled was influenced by both the instruction and the cueing manipulations, as would be expected. (b) The imagery and verbal instructions resulted in more clustering and in higher IPG scores, both of which reflect the amount of within-group organization. but instruction conditions did not influence the NGR scores as a main effect. (c) The NGR scores were influenced primarily by the cueing manipulation. The 20 -group cues condition yielded recall of a greater number of input groups, and this effect increased across trials. The cueing variable did not influence either the clustering or IPG measures as a main effect.

\section{DISCUSSION}

The major findings of the present study were: (a) Instructions to use image mediators produced higher recall than instructions to use sentence mediators, which in turn produced higher recall than control instructions. (b) When within-group organization was high enough. the 20-group cues condition produced an increase in recall.

The first result in part replicates the Mueller and 
Jablonski (1970) data, but these Es found no consistent difference between sentence mediation and control instructions. These differing results may have been due to procedural differences. In the Mueller and Jablonski study, the words were presented singly with a varied order across trials, while in the current study, the stimuli were shown in consistent four-word groups, a procedure which presumably facilitated the use of any mediational technique. Also, the Mueller and Jablonski study used a mixed high- and low-imagery list, while the current list contained only high-imagery words.

The clustering and IPG data indicate that the two kinds of learning instructions facilitated recall by increasing within-group organization. Similarly, the imagery instructions seem to have been more effective than the verbal instructions because of the greater within-group cohesiveness produced by the former. These conclusions fit well with an organizational account of free recall learning (e.g., Tulving, 1966) and with previous attempts to explain the beneficial effects of imagery as a mediational device. For instance, Tulving: McNulty, and Ozier (1965) found that free recall and subjective organization were greater for words high in "vividness" than for those low in vividness. (Vividness was defined in a manner similar to measures of imagery.) Tulving et al attributed the superior performance on the high-vividness words to the greater ease with which these stimuli could be organized into groups. The present data also agree with Bower's (1970a) account of the role of image mediators in paired-associate learning. Bower found that instructions to use images in which the stimulus and response terms were interacting resulted in higher associative recall than instructions emphasizing either separate images for the stimulus and response terms or rote learning. There was no difference among the groups in stimulus-term recognition. Bower concluded that the facilitation effect produced by high imagery for paired-associate learning is due to the ease with which associative relationships are formed rather than to increased reliability of stimulus encoding or to increased stimulus distinctiveness.

The locus of the differential effect of imagery vs verbal instructions seems, then, to be in the strength of within-group organization. Exactly why images are superior in this respect is not established by our data. Images may provide an additional code. or they may have "spatially parallel" properties that decrease memory load or increase ease of retrieval (Paivio. 1971). None of these possibilities is ruled out by the data. Further. it is possible that the difference between the imagery and verbal conditions was due to the greater ease of production of images as compared to sentences rather than to differences in the inherent properties of the two kinds of mediators. The formation of a sentence with four nouns may be more difficult than the formation of an image involving four objects.

The finding of a positive effect of cueing in the 20-group cues condition is important because it demonstrates that lists containing preexperimental relations among the items are not unique in this respect. As has been pointed out a number of times (e.g. Postman, 1972; Slamecka, 1968, 1969), it is a natural prediction from an organizational theory of free recall that cueing with lis: items should be beneficial to recall. This is because organizational theory posits that free recall learning entails the formation of higher-order units (i.e., it posits that storage of items is "dependent") (Slamecka, 1968, 1969). Therefore, each word should be an effective cue for its unit, and presenting some list items at recall should facilitate recall of other list items. The fact that this prediction has repeatedly not been supported with lists of unrelated words has been troublesome for an organizational account of free recall and has resulted in various at tempts to explain away the negative findings by positing sources of bias against showing a positive cueing effect. The present data argue that at least some of the reasons previously given for the negative results are valid: our results show that if the conditions are right, a positive cueing effect is obtained even with unrelated words.

Finally, it may be worthwhile reiterating the finding that the learning instructions mainly affected within-group organization and had little effect on group retrievability; the opposite was true of the cueing variable. These results support what seems to be an assumption (often implicit) of much of the work in free recall, namely, that the formation of higher-order units and the development of a retrieval plan are distinct processes influenced by different variables.

\section{REFERENCES}

Allen, M. M. Cueing and retrieval in free recall. Journal of Experimental Psychology, 1969, 81, 29-35.

Bower. G. H. Imagery as a relational organizer in associative learning. Journal of Verbal Learning \& Verbal Behavior. 1970a, 9. 529-533.

Bower. G. H. Organizational factors in memory. Cognitive Psychology, 1970b, 1, 18-46.

Bower, G. H.. Lesgold, A. M., \& Tieman, D. Grouping operations in free recall. Journal of Verbal Learning \& Verbal Behavior. $1969,8,481493$.

Bower, G. H., \& Winzenz, D. Comparison of associative learning strategies. Psychonomic Science, 1970. 20, 119-120.

Hudson. R. L., \& Austin. J. B. Effect of context and category name on the recall of categorized word lists. Journal of Experimental Psychology. 1970.86.43-47.

Mueller. J. H.. \& Jablonski. E. M. Instructions, noun imagery, and priority in free recall. Psychological Reports, 1970, 27. 559-566.

Paivio, A. Imagery and rerbal processes. New York: Holt, Rinehart \& Winston. 1971

Paivio, A.. \& Foth, D. Imaginai and verbal mediators and noun concreteness in paired-associate learning: The elusive interaction. Journal of Verbal Learning \& Verbal Behavior. $1970,9.384 .390$.

Paivio, A.. Yuille. J. C.. \& Madigan. S. A Concreteness, imagery. and meaningfulness values for 925 nouns. Journal of Experimental Psychology Ionograph Supplements. 1968. 76(1. Pt. 2$).$

Postman, L. A prasmatic liew of organization theory. In $E$. 
Tulving and W. Donaldson (Eds.), Organization of memory. New York: Academic Press, 1972.

Roenker, D. L., Thompson, C. P., \& Brown, S. C. Comparison of measures for the estimation of clustering in free recall. Psychological Bulletin, 1971, 76, 45-48.

Slamecka, N. J. An examination of trace storage in free recall. Journal of Experimental Psychology, 1968, 76, 504-513.

Slamecka, N. J. Testing for associative storage in multitrial free recall. Journal of Experimental Psychology, 1969, 81, $557-560$.

Slamecka, N. J. The question of associative growth in the learning of categorized material. Journal of Verbal Learning \& Verbal Behavior, 1972, 11, 324-332.

Tulving, E. Subjective organization and effects of repetition in multitrial free-recall learning. Journal of Verbal Learning \& Verbal Behavior, 1966, 5, 193-197.

Tulving, E., McNulty, J. A., \& Ozier, M. Vividness of words and learning to learn in free-recall learning. Canadian Journal of Psychology, 1965, 19, 242-252.

Tulving, E., \& Pearlstone, Z. Availability versus accessibility of information in memory for words. Journal of Verbal Learning \& Verbal Behavior, 1966, 5, 381-391.

Wood, G. Retrieval cues and the accessibility of higher-order memory units in multi-trial free recall. Journal of Verbal Learning \& Verbal Behavior, 1969, 8, 782-789.

\section{NOTES}

1. ARC is corrected for the expected number of consecutive words from the same input group and is defined so that chance clustering is set at zero and perfect clustering equals one.

2 . It is debatable whether the 5 -group cues or the no cues condition is the appropriate control for showing a positive effect of cueing in the 20-group cues condition. The 5-group cues condition controls for the time required to read the cues in the 20-group cues condition, but it probably entails an additional disruption effect because the cues do not in fact provide clues to any of the other words (assuming that the input groups have provided the basis for organization of the words). Therefore, if the 5-group cues condition were taken as the baseline condition, any positive effect of cueing in the 20-group cues condition would probably be overestimated. If, however, the no cues condition is used as the baseline condition, no control is provided for the time to read the cues, and any positive effect of cueing in the 20-group cues condition is probably underestimated. The latter, conservative approach is adopted here.

(Received for publication July 20, 1973; accepted August 17, 1973.) 